\title{
VSEM: From Technology Demonstrator Towards Integrated Educational Tool
}

\author{
NHM Caldwell, BC Breton, DM Holburn, and RP Robertson.
}

Department of Engineering, Cambridge University, Trumpington Street, Cambridge CB2 1PZ, UK

In our previous work, we developed VSEM (Virtual Scanning Electron Microscope) as a response to the clear need to provide training in microscopy theory and practice to university students, occasional instrument users, and novice operators [1]. The VSEM application combines a software simulation of the operation and behaviour of the SEM with a virtual reality model of the instrument, implemented using Java, QuickTime, and VRML (Virtual Reality Markup Language) [2].

Recent research and development of the VSEM has been aimed at addressing technical and pedagogical aspects of the existing application. In the existing system, most of the emulation controls (for focus, astigmatism, etc.) simulate the appropriate instrument aspect through mouseoperated sequences of pre-generated images. In addition to the drawback of requiring many images to be captured per individual specimen, this method does not allow arbitrary multiple actions to be performed consecutively and cumulatively on a specific image frame. That is, if the user adjusts the contrast of a simulated specimen, and then attempts to adjust the brightness, the original contrast is restored, rather than the brightness change being performed on the image at the new contrast setting.

At the time of our original investigations, the dynamic generation of image sequences in real-time using image processing algorithms was computationally impracticable. Recent advances in the processing power of standard personal computers render certain procedures now practical. By combining the facilities found in the Java 2D API (from Sun Microsystems) and the Java Image Processing Library (from JH Labs), a relatively complete set of image processing functions could be incorporated into test applets. Experimentation confirmed that convolving images with spatial image filters, derived from Gaussian kernels and other functions, could effectively simulate focus and astigmatism. On a real SEM, functions such as brightness may be set between 0 and 100\% with a precision of $0.1 \%$ or better. For simulation purposes, setting ten discrete levels between the maximum and minimum values for a control represents an appropriate compromise between verisimilitude and computational limits.

In the current prototype, each simulated specimen can be manipulated in terms of magnification, focus, astigmatism, brightness, and contrast. For controls other than magnification, a series of filters is invoked to produce the required settings. Thus if the user chooses to adjust the brightness of a specific specimen, the complete sequence of varying brightness levels for the specimen at the current magnification, focus, astigmatism, and contrast levels is generated first, and the user may then increase or decrease brightness repeatedly without inducing severe detail loss through overapplication of the filters to the currently displayed images. Magnification is a special case, as distinct images are needed for each level of magnification to avoid pixellation artefacts.

Image sequence generation takes variable amounts of time depending on how far the final images are from the baseline specimen images. On a Pentium III $533 \mathrm{MHz}$ processor with 128 megabytes of RAM, the maximum delays for creating 512-square sequences ranged from 15 to 80 seconds. These delays were still undesirably long. The SEM itself suggests a solution by analogy to the reduced 
raster mode of many modern instruments. A smaller central section of the VSEM display is designated as the working display with control adjustments only being reflected in changes to that area (and hence substantially reducing the computation time) until the user is satisfied, whereupon the entire VSEM display is updated. Image sequences could now be computed with maximum delays ranging from 5 to 15 seconds. As computer hardware continually improves, these delays will be reduced further.

Although the original VSEM application had some on-line help facilities and support documentation, these concentrated on the technical specifics of installing and operating the software itself, rather than explaining the theory and practice of scanning electron microscopy. Cambridge University Engineering Department offers a final-year undergraduate course entitled "VLSI Design", which includes a practical session on the use of the SEM for integrated circuit testing. We decided to redevelop these on-line help facilities into web-based courseware to support departmental undergraduate teaching.

In developing web-based resources, there is a dichotomy between usability on all platforms and support for rich interactivity. As many aspects of the SEM would benefit from dynamic explanations, we have preferred highly interactive solutions, choosing Macromedia's Director package as the authoring tool. Director can combine text, images, graphical simulations, audio and video clips into a cohesive whole. Director files may be substantially compressed, enabling delivery of course materials via Internet Explorer and Netscape browsers for PCs and Macintosh computers using the freely available Shockwave plug-in package.

The resulting SEM courseware provides an overview of the instrument with material on the basics of columns, electron guns, signal detection methods and contrast mechanisms. Text and images are supplemented with a running audio commentary and graphical simulations of difficult concepts such as electron lenses and signal generation. A basic virtual SEM was also implemented within the lectures (as a Director "movie") to demonstrate magnification, sample movement, brightness, contrast, focusing, and the distinction between secondary and backscattered electron images.

Ongoing work is concentrating on the development of a Microscopy Encyclopaedia to complement the VSEM environment. This will explore instrument theory and practice at a more detailed level than the overview lecture. Interactive simulations are being developed using Director for each SEM subsystem with the aim of engaging the student in absorbing and understanding the material. It is hoped also to construct 3-D models to allow users to explore the instrument's internals.

Future work will include evaluating the strategies employed so far in the VSEM project to determine which techniques to pursue in progressing VSEM towards an integrated educational application [3].

References

[1] DM Holburn et al. Microscopy \& Analysis, March/April 2000, 5

[2] DM Holburn et al. Microscopy and Microanalysis, 6 (s2) (2000), 1156.

[3] NHM Caldwell and BC Breton would like to acknowledge the financial support of LEO Electron Microscopy Ltd. and the Isaac Newton Trust. The authors would like to thank Paul Boyle, Dan Lamping and Geoff Martin for their work in furthering the VSEM project. 